\title{
Novel dehydroepiandrosterone troche supplementation improves the serum androgen profile of women undergoing in vitro fertilization
}

This article was published in the following Dove Press journal:

Drug Design, Development and Therapy

9 October 2015

Number of times this article has been viewed

\author{
Kevin N Keane ${ }^{1,2}$ \\ Peter M Hinchliffe ${ }^{2}$ \\ Navid Namdar ${ }^{2}$ \\ Jason L Conceicao ${ }^{2}$ \\ Philip Newsholme' \\ John L Yovich ${ }^{1,2}$ \\ 'School of Biomedical Sciences, \\ Curtin Health Innovation Research \\ Institute - Biosciences, Curtin \\ University, ${ }^{2}$ PIVET Medical Centre, \\ Perth, Australia
}

\begin{abstract}
Dehydroepiandrosterone (DHEA) is the most abundant steroid hormone in the circulation and has potent multifunctional activity. Epidemiological evidence suggests that levels of serum DHEA decrease with advancing age, and this has been associated with onset or progression of various age-related ailments, including cognitive decline and dementia, cardiovascular disease, and obesity. Consequently, these findings have sparked intense research interest in DHEA supplementation as an "antiaging" therapy. Currently, DHEA is being used by $25 \%$ of in vitro fertilization (IVF) clinicians as an adjuvant in assisted reproductive programs, yet the therapeutic benefit of DHEA is unclear. Here, we examined the use of novel DHEAcontaining oral troches in patients undertaking IVF and investigated the impact of these troches on their serum androgen profile. This retrospective study determined the androgen profile of 31 IVF patients before (baseline) and after DHEA supplementation (with DHEA). Baseline serum measurements of testosterone (total and free), DHEA sulfate (DHEAS), sex hormonebinding globulin (SHBG), and androstenedione were made before and after supplementation. Each patient received DHEA troches containing $25 \mathrm{mg}$ of micronized DHEA, and troches were administered sublingually twice daily for a period of no greater than 4 months. Adjuvant treatment with DHEA boosted the serum concentration of a number of androgen-related analytes, including total and free testosterone, androstenedione, and DHEAS, while serum SHBG remained unchanged. Supplementation also significantly increased the free-androgen index in IVF patients. Interestingly, the increase in serum analyte concentration following DHEA supplementation was found to be dependent on body mass index (BMI), but not individual age. Patients with the lowest BMI $\left(<20.0 \mathrm{~kg} / \mathrm{m}^{2}\right)$ tended to have lower testosterone and DHEAS, but higher SHBG and androstenedione levels in comparison with other BMI groups postsupplementation. However, patients in the highest BMI group $\left(>30.0 \mathrm{~kg} / \mathrm{m}^{2}\right)$ tended to have lower androgen responses following DHEA supplementation, but these were not statistically different from the corresponding baseline level. This method of DHEA administration results in a similar enhancement of testosterone, DHEAS, and androstenedione levels in comparison with other methods of administration. Furthermore, we showed that BMI significantly influences DHEA uptake and metabolism, and that BMI should be carefully considered during dosage calculation to ensure a significant and robust androgen-profile boost.
\end{abstract}

Keywords: androstenedione, testosterone, sex hormone-binding globulin, IVF, oral troches, drug delivery

\section{Introduction}

Dehydroepiandrosterone (DHEA) is a multifunctional adrenal prohormone that has potent biological activity. It is the most abundant steroid hormone in the circulation, but natural serum concentrations depend on individual age and sex. ${ }^{1}$ DHEA can directly 
elicit its effects through interaction with receptors located in the plasma membrane, cytoplasm, and nuclear membrane, which ultimately translocate inside the nucleus, where they regulate gene expression. ${ }^{1,2}$ It has been reported that DHEA is a potent ER $\beta$ agonist, ${ }^{3}$ but a weak AR agonist. Although DHEA is known to associate with other important cellsignaling and metabolic receptors, such as PPAR $\gamma$, a specific DHEA receptor has not yet been identified. ${ }^{2}$ Conversely, some earlier studies have indicated that DHEA can bind membranebound protein complexes that may relay DHEA-induced processes. ${ }^{4}$ However, DHEA primarily indirectly stimulates various downstream biological responses, by functioning as a precursor for the synthesis of powerful sex-steroid hormones (intracrinology), including testosterone and estradiol. ${ }^{1}$ Therefore, DHEA supplementation is believed to offer several advantages over testosterone administration, due to direct albeit weak effects on the AR and as a precursor for other androgens, which ultimately results in androgen synthesis when required, rather than flooding biological systems directly with active androgens, which could cause side effects. ${ }^{5}$

Metabolic production of DHEA occurs from the substrate 17-hydroxypregnenolone, under the action of the cytochrome P450c17, which is highly expressed in the adrenal glands. ${ }^{1,6}$ In downstream peripheral tissues, DHEA can be converted to androstenedione and subsequently testosterone by the action of $3 \beta$-HSD and $17 \beta-H S D$, respectively. ${ }^{1}$ Alternatively, DHEA-derived androstenedione can be converted to estrogen via P450 aromatase activity, demonstrating an important role for DHEA in hormone production for both sexes. DHEA mainly circulates in the vasculature as an inactivated sulfated derivative (DHEAS). ${ }^{7}$ A balance exists between these DHEA structures, and the interconversion between the two is dependent on sulfotransferase and steroid sulfatase enzyme activity in peripheral tissues. Since DHEAS is the major form of DHEA present in the circulation (typically $10 \mu \mathrm{M}$ DHEAS to $30 \mathrm{nM}$ DHEA), ${ }^{8,9}$ DHEAS is routinely quantified in the clinic as an indirect measure of DHEA status.

Epidemiological evidence suggests that levels of serum DHEA/DHEAS decrease with advancing age, ${ }^{10}$ and reduced concentrations have been associated with onset or progression of various age-related ailments, including cognitive decline and dementia, ${ }^{11,12}$ cardiovascular disease, ${ }^{13}$ cancer, ${ }^{14}$ obesity, ${ }^{15}$ and autoimmune disease. ${ }^{1,16}$ However, in many of these fields of research, the precise role of DHEA in mediating disease is not clear, and in most cases, the literature also demonstrates opposite and inconsistent relationships, which further complicates the function of DHEA. ${ }^{13,14,17,18}$ Nonetheless, these studies and others have sparked intense research interest in DHEA supplementation as an "antiaging" therapy, but DHEA use remains controversial. , $^{7,8}$

Within the assisted reproductive technology field, eg, in vitro fertilization (IVF), the primary cause of reduced success rates is increasing female age. Interestingly, this is closely associated with an age-induced reduction of ovarian reserve, a measure of reproductive potential of the ovary. In Western cultures, there is an increasing propensity of females to delay childbirth as a result of socioeconomic factors, particularly in relation to extended education and dedication to careers, which is unfavorable for their ovarian reserve. Furthermore, female androgen levels are vastly lower than in the male (as a component part of sex phenotypic differences), but good biological androgenic function is thought to be required for successful reproduction, as these hormones are believed to modulate ovarian physiology, including oocyte and follicle maturation, receptor expression, DNA repair, mitochondrial function, and the induction of local effects on the endometrium during ovulation and implantation. ${ }^{19-22}$ Consequently, these findings have led to the recent use of DHEA as an adjuvant in assisted reproductive technology programs, ${ }^{22}$ particularly for older patients, who may have reduced androgen levels or are categorized as poor ovarian responders according to the Bologna criteria. ${ }^{23}$ However, although there is some evidence to indicate improvement in IVF success rates with DHEA, ${ }^{24-28}$ its use remains controversial, and several clinical trials and meta-analyses have so far failed to reveal convincingly any true therapeutic benefit of DHEA adjuvant treatment in IVF cycles. ${ }^{19,22,29}$ This is due to inconsistent results describing outcomes, due to small or poorly designed trials that are self-controlled.

Most of the DHEA adjuvant treatments used in IVF have utilized oral (tablet) routes of DHEA administration. 25,27,29,30 Herein, we describe the unique concept of supplementing female IVF patients with novel, lipid-based DHEA-containing oral sublingual troches to bypass first-pass metabolism, as DHEA is highly metabolized and inactivated by the liver. To the best of our knowledge, this is the first study of its kind to report the administration of DHEA via oral troches, and the first report demonstrating an increase in the androgenic profile in response to DHEA therapy for female patients undergoing IVF.

\section{Materials and methods DHEA troche manufacture}

DHEA troches were prepared according to an in-house recipe. Briefly, DHEA microparticles were dispersed in a sweetened and flavored mix of polyethylene glycol base $(48-105 \mu \mathrm{M})$. This mix allows DHEA to release slowly and uniformly sublingually. This in turn allows better absorption 


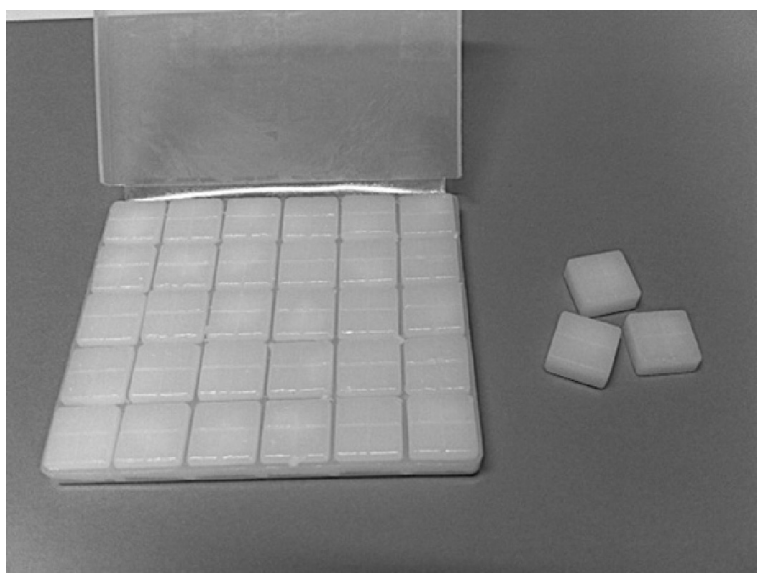

Figure I Novel dehydroepiandrosterone (DHEA) troches. Note: Photograph displaying the novel DHEA-containing oral troches designed by our group, cross-scored to enable fractional dosages from $12.5 \mathrm{mg}$ to $50 \mathrm{mg}$.

of DHEA through the buccal route. There were 30 troches per preparation tray (Figure 1), with each troche containing $50 \mathrm{mg}$ DHEA. Each troche was cross-scored, enabling an easy cut to provide $25 \mathrm{mg}$ per half, or even $12.5 \mathrm{mg}$ per quarter.

\section{Patients and troche administration}

This was a retrospective study (January 2012 to April 2014) examining the serum androgen profile of 31 poor-prognosis IVF female patients before (baseline) and after DHEA supplementation, using novel DHEA-containing oral troches designed by our group. Patients were not assigned to any other adjuvant treatment during this period. Serum measurements of testosterone (total and free), DHEAS, sex hormone-binding globulin (SHBG), and androstenedione were made before (baseline) and 4 weeks after supplementation (treated). For supplementation, each patient utilized half a DHEA troche containing $25 \mathrm{mg}$ of micronized DHEA sublingually twice daily for a period of 6 weeks to a maximum of 4 months. At the fourth-week review, only one patient in this series had a troche reduction to $12.5 \mathrm{mg}$ because of an excess testosterone level; two others reported mild acne, which did not require changes in DHEA administration. As a comparative control, the androgen profile of patients of similar age (Figure 2A) and body mass index (BMI; Figure 2B) were also assessed on two separate occasions to determine spontaneous change in hormone levels (Figure 3). In these patients, the second measurement (measurement 2 ) was made on average $50 \pm 3$ weeks (standard error of mean) following the initial androgen-profile screen (Figure 3).

\section{Androgen assays}

Analysis of serum testosterone, SHBG, and DHEAS was performed using a Cobas 8000 modular analyzer (Hoffman-La Roche Ltd, Basel, Switzerland) with coefficients of variation of less than $3.14 \%, 2.23 \%$, and $3.06 \%$, respectively. Analysis of serum androstenedione was performed using the Immulite 2000 immunoassay system (Siemens AG, Berlin, Germany) with a coefficient of variation of less than $5.23 \%$. Error bars represent \pm standard error of mean.

\section{Statistical analysis}

Statistical analysis of the data was undertaken using GraphPad Prism version 6.0 (GraphPad Software Inc., La Jolla, CA, USA). Multiple comparisons were made for each measured variable using one-way analysis of variance and applying Tukey's test. When the data were grouped according to age or BMI, multiple comparisons were analyzed using two-way analysis of variance and applying Tukey's test. Data was considered statistically different if $P<0.05$.

\section{Ethical considerations}

PIVET is accredited with both the self-regulatory national Australian authority of the Reproductive Technology Accreditation Committee, as well as the statutory Western
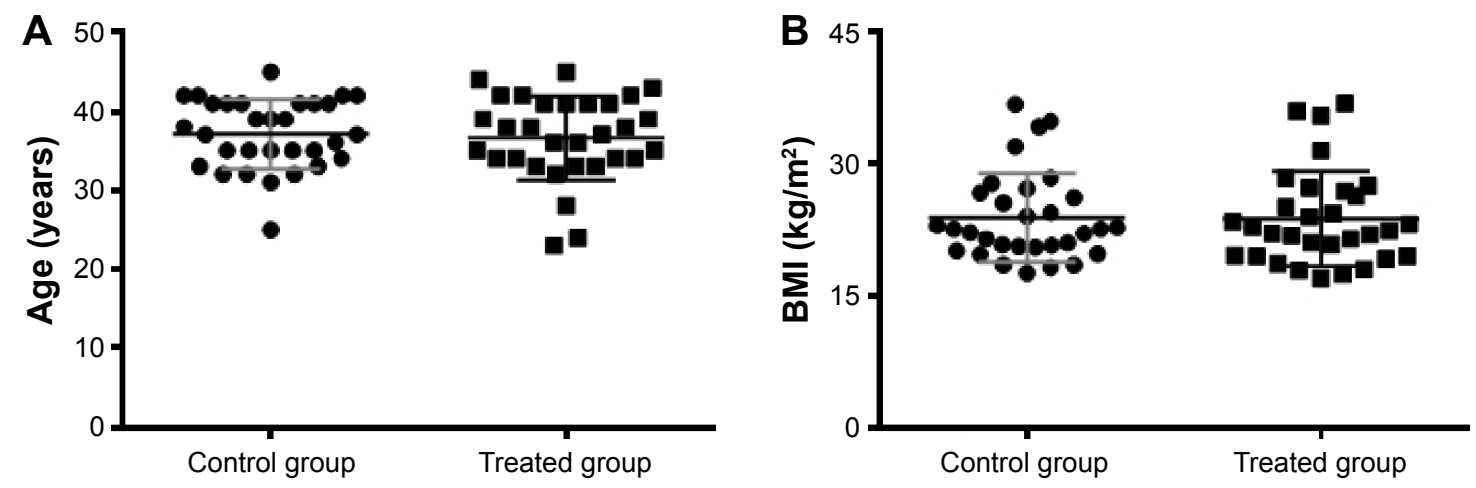

Figure 2 Patient age and body mass index (BMI) characteristics.

Note: Patients in each cohort either not receiving dehydroepiandrosterone (DHEA) troches (control group; circles) or receiving DHEA troches (treated group; squares) had similar age $(\mathbf{A})$ and $\mathrm{BMI}(\mathbf{B})$ means and ranges. 
A

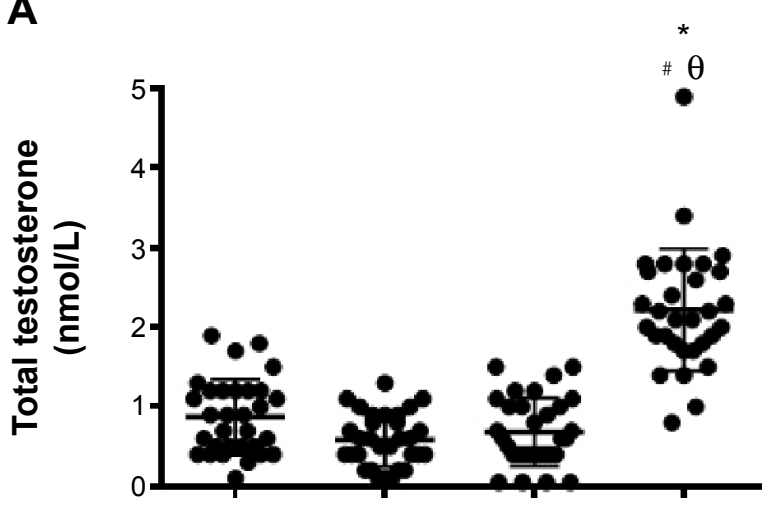

C

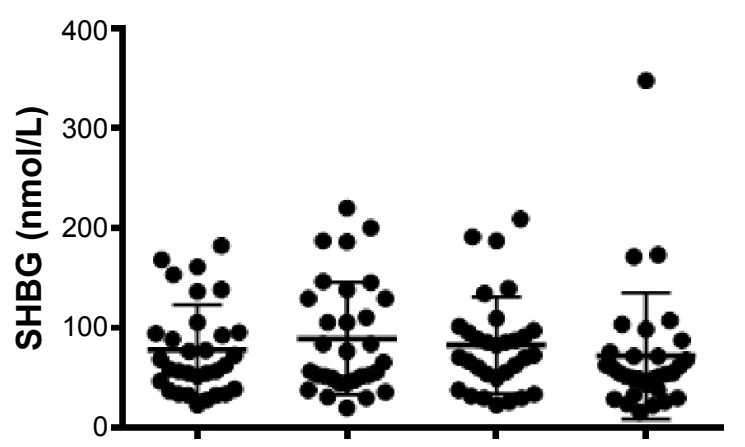

E

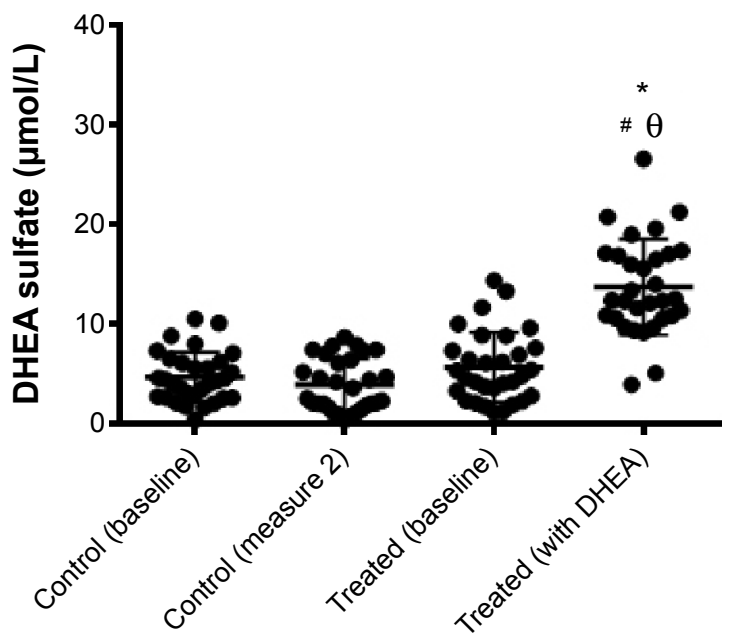

B

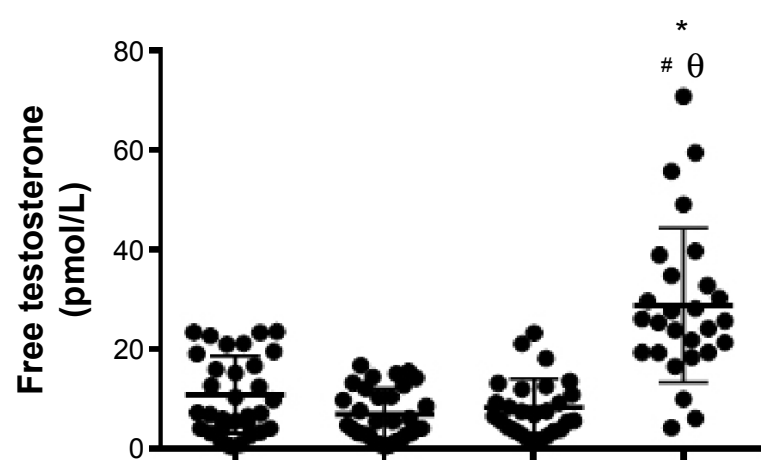

D

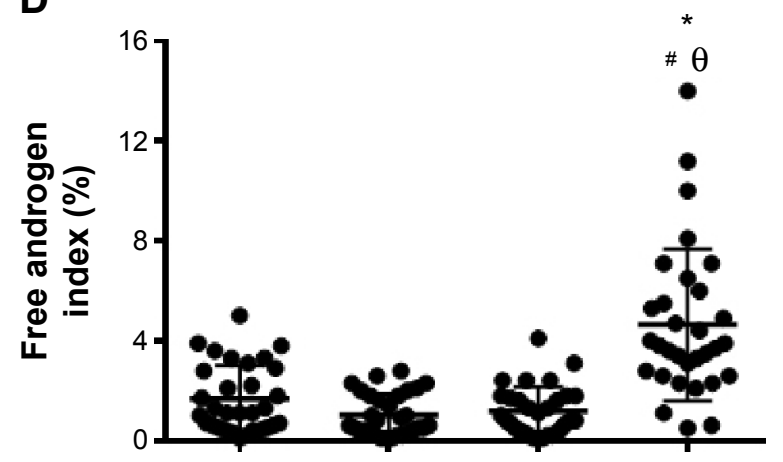

$\mathbf{F}$

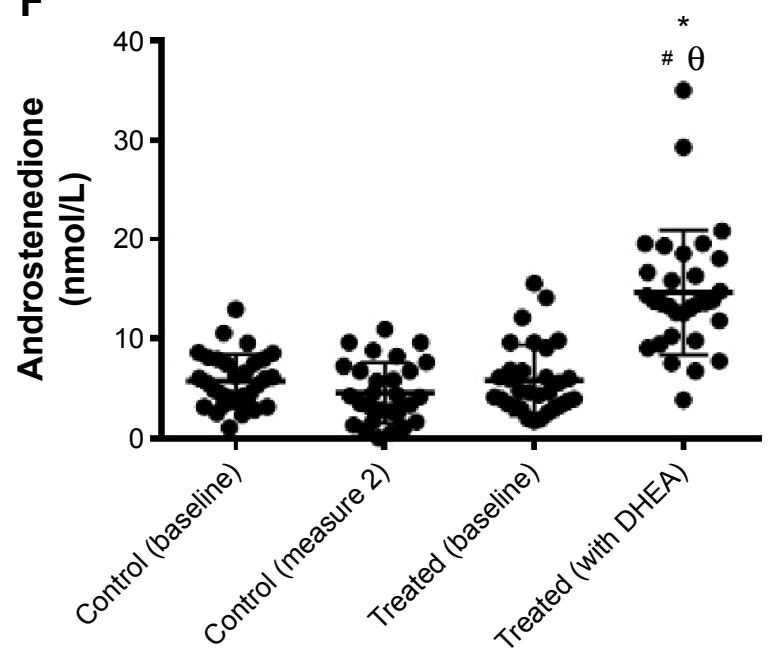

Figure 3 Effect of dehydroepiandrosterone (DHEA) troches on serum androgen profile.

Notes: Treatment with DHEA troches increased the serum concentration of a number of androgen-related analytes. Total/free testosterone (A, B), free-androgen index (D), DHEA sulfate (E), and androstenedione (F) were all significantly increased. However, sex hormone-binding globulin (SHBG) remained unchanged (C). The control cohort did not receive DHEA, and measure 2 was made on average $50 \pm 3$ weeks (standard error of mean) following the initial androgen-profile screen. Significantly different from *control (baseline), "control (measure 2), and ${ }^{\theta}$ treated (baseline).

Australian state accreditation body of the Reproductive Technology Council of Western Australia (established under the Western Australian Human Reproductive Technology Act, 1991). These agencies monitor all activities conducted at PIVET and demand oversight by a National Health and Medical Research Council-constituted institutional ethics committee who endorse all clinical and laboratory protocols. PIVET laboratories are also accredited with the National Australian Testing Authority, which requires strict adherence to quality-assurance protocols. Specific ethics approval was not required for this study, as all procedures and blood tests were embraced by routine approved clinical protocols. 
However, reporting of the data was approved under Curtin University Ethics Committee RD_25-10 general approval for retrospective data analysis 2015. All participants signed written consent forms stating that:

The data will be used for national statistical reporting, regulatory review and population based research, and there is no publication of information that identifies any individual.

\section{Results}

\section{The effect of DHEA troches on serum androgen profile}

Adjuvant treatment with DHEA troches increased serum androgen profile. Total and free testosterone (Figure 3A and B) were both significantly increased in patients treated with DHEA: from mean values of $0.68 \pm 0.08 \mathrm{nmol} / \mathrm{L}$ and $8.2 \pm 1.1 \mathrm{pmol} / \mathrm{L}$, respectively, to $2.2 \pm 0.14 \mathrm{nmol} / \mathrm{L}$ and $29.0 \pm 3.0 \mathrm{pmol} / \mathrm{L}$, respectively. Serum SHBG remained unchanged (Figure 3C), but the free-androgen index (FAI) was significantly elevated from $1.2 \pm 0.18$ at baseline to $4.6 \pm 0.54$ postsupplementation (Figure 3D). Both DHEAS (Figure 3E) and androstenedione (Figure 3F) were significantly increased: from $5.7 \pm 0.63 \mu \mathrm{mol} / \mathrm{L}$ and $5.9 \pm 0.63 \mathrm{nmol} / \mathrm{L}$, respectively, to $13.7 \pm 0.87 \mu \mathrm{mol} / \mathrm{L}$ and $14.7 \pm 1.12 \mathrm{nmol} / \mathrm{L}$, respectively, after patients used DHEA troches. The comparative control group demonstrated a slight reduction in total and free testosterone, FAI, DHEAS, and androstenedione, but this was not statistically significant. No significant change in SHBG was observed in this group.

\section{The influence of age on DHEA-induced serum androgen profile}

When the androgen responses to DHEA were stratified according to female age, the increase in serum analyte concentration was found to be independent of the age of women receiving DHEA troches. As described earlier, supplementation caused a statistically significant increase in total and free testosterone (Figure 4A and B, respectively), increased DHEAS and androstenedione levels (Figure 4C and D, respectively) in comparison with baseline measurements, but no significant alteration in SHBG was observed (Figure 4E). Interestingly, when the fold change between baseline and DHEA-supplemented values were calculated for testosterone, DHEAS, and androstenedione within each individual age-group, the relative increase in serum concentration was similar across all age-groups and for each analyte type (Figure $4 \mathrm{~F}$ ). This indicated that the troches induced the same fold increase in androgen profile regardless of age.

\section{The influence of body mass index on DHEA-induced serum androgen profile}

When the androgen responses to DHEA were separated according to female BMI, the increases in serum analyte concentration from DHEA supplementation were found to be dependent on BMI. Patients in the $20.1-25.0 \mathrm{~kg} / \mathrm{m}^{2}$ BMI category displayed a statistically significant increase in total and free testosterone (Figure 5A and B, respectively), increased DHEAS and androstenedione levels (Figure 5D and $\mathrm{E}$, respectively) in comparison with baseline levels, but no significant change in SHBG was observed (Figure 5C). Those with a BMI between 25.1 and $30.0 \mathrm{~kg} / \mathrm{m}^{2}$ exhibited the same response, but the elevation in androstenedione was not significantly different from the corresponding baseline measurement (Figure 5E). Patients in the lowest BMI group $\left(<20.0 \mathrm{~kg} / \mathrm{m}^{2}\right)$ tended to have lower testosterone and DHEAS (Figure 5A, B, and D), but higher SHBG and androstenedione (Figure 5C and $\mathrm{E}$ ) levels in comparison with other BMI groups postsupplementation. However, the supplement-induced enhancement of free testosterone was not significantly different from the corresponding baseline measurement (Figure 5B). Most interestingly, patients in the highest BMI group $\left(>30.0 \mathrm{~kg} / \mathrm{m}^{2}\right)$ also tended to have lower androgen responses to DHEA supplementation in comparison with other BMI groups. Although there was a trend toward increased levels of testosterone (Figure 5A and B), DHEAS (Figure 5D), and androstenedione (Figure 5E) after administration of DHEA troches, the increase was not statistically different from the corresponding baseline level.

\section{Discussion}

DHEA supplementation is now widely used by more than $25 \%$ of IVF clinicians worldwide for poor-responding patients with diminished ovarian reserve, yet the true benefit of this adjuvant therapy remains unknown. ${ }^{19}$ In addition, most original clinical articles and meta-analyses discussing the application of DHEA therapy in IVF have failed to report the impact of supplementation on the level of related serum androgens in the bloodstream, with the notable exception of Zhang et al. ${ }^{31}$ In the current study, we attempted to address this issue and demonstrate that the use of novel DHEAcontaining oral troches could enhance the serum androgen profile significantly in patients undergoing IVF.

We demonstrated that sublingual DHEA administration (50 mg daily) increased free testosterone, total testosterone, 



Figure 4 Influence of age on dehydroepiandrosterone (DHEA)-induced serum androgen profile.

Notes: The increase in serum analyte concentration was found to be independent of the age of women receiving DHEA troches. Supplementation caused a statistically significant increase in total and free testosterone (A and B, respectively), increased DHEA sulfate (DHEAS) and androstenedione levels (C and D, respectively), but no significant alteration in sex hormone-binding globulin (SHBG) was observed (E). When the fold change between baseline and DHEA-supplemented values were calculated for testosterone, DHEAS, and androstenedione for each of the individual age-groups, the relative increase in serum concentration was not significantly different with regard to age or analyte type $(\mathbf{F})$. *Significantly different from baseline measurements of patients aged $<35$ years $(P<0.05)$; ${ }^{*}$ significantly different from baseline measurements of patients aged 35-39 years $(P<0.05)$; ${ }^{\theta}$ significantly different from baseline measurements of patients aged $\geq 40$ years $(P<0.05)$.

androstenedione, and DHEAS levels in a highly significant manner $(P<0.0001)$. No change in SHBG was detected, but the largest rise was observed in FAI, with a 3.8-fold increase. This was followed by an increase in free and total testosterone (3.5-fold and 3.3-fold, respectively), and then androstenedione (2.5-fold) and DHEAS (2.5-fold). These data are comparable to other reports showing that oral administration of DHEA (50 mg daily) in older women (40-70 years old) increased the levels of androstenedione and total testosterone by approximately twofold, which is of a similar magnitude to those observed in our current study. ${ }^{32}$ Conversely, in another study, the increment of androstenedione and total testosterone levels in menopausal female patients (60-70 years old) following topical administration of DHEA ( $2 \mathrm{~g} /$ daily) was 


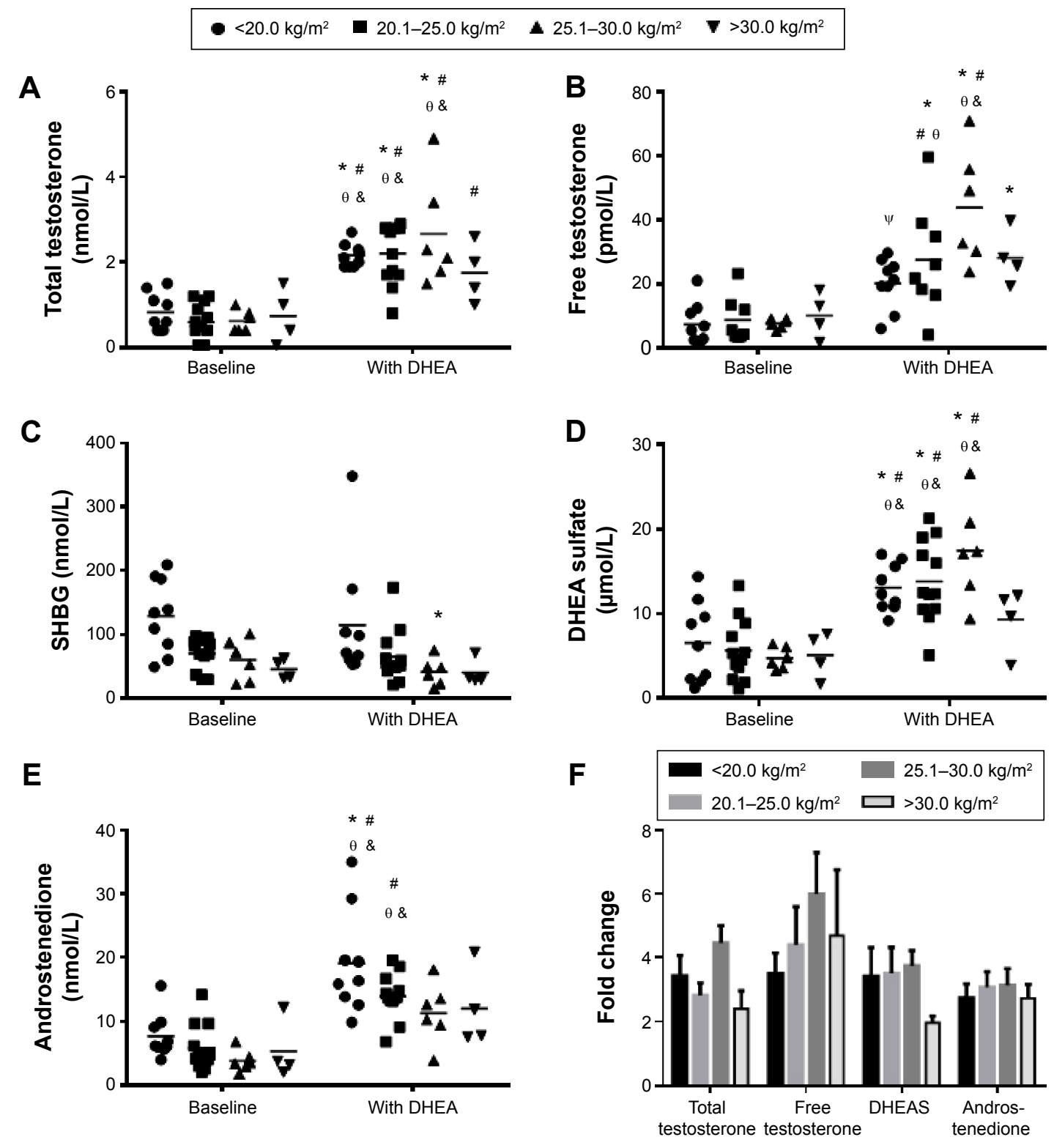

Figure 5 Impact of body mass index (BMI) and dehydroepiandrosterone (DHEA) on serum androgen profile.

Notes: DHEA increased serum androgen content in a BMI-dependent manner. Patients in the middle BMI categories displayed a significant increase in total and free testosterone (A and B, respectively) and increased DHEA sulfate (DHEAS) and androstenedione levels (D and E, respectively). No significant change in sex hormone-binding globulin (SHBG) was observed $(\mathbf{C})$. Lower-BMl patients $\left(<20.0 \mathrm{~kg} / \mathrm{m}^{2}\right)$ tended to have lower testosterone and DHEAS (A, B, D), but higher SHBG and androstenedione (C and E) levels in comparison with other BMl groups postsupplementation. Those in the highest-BMl group $\left(>30.0 \mathrm{~kg} / \mathrm{m}^{2}\right)$ also tended to have lower androgen responses to DHEA supplementation in comparison with other BMl groups. Although there was a trend toward increased levels of testosterone (A and B), DHEAS (D), and androstenedione (E) after administration of DHEA troches, the increase was not statistically different from the corresponding baseline levels. The fold change for each androgen in each BMl group is shown in (F). *Significantly different from baseline measurements of patients $<20.0 \mathrm{~kg} / \mathrm{m}^{2}(P<0.05)$; ${ }^{*}$ significantly different from baseline measurements of patients $20.1-25.0 \mathrm{~kg} / \mathrm{m}^{2}(P<0.05)$; ${ }^{\theta}$ significantly different from baseline measurements of patients $25.1-30.0 \mathrm{~kg} / \mathrm{m}^{2}(P<0.05)$; ${ }^{8}$ significantly different from DHEA measurements of patients $>30.0 \mathrm{~kg} / \mathrm{m}^{2}(P<0.05) ;{ }^{*}$ significantly different from patients with DHEA and BMl of $25.1-30.0 \mathrm{~kg} / \mathrm{m}^{2}(P<0.05)$.

twofold and 1.5-fold, respectively, which was significantly lower than that shown in our current study. ${ }^{33}$ Furthermore, we found that DHEAS was increased by approximately 2.5 -fold using oral troches, while DHEAS was increased by a similar 2.3 -fold in this previous percutaneous study. ${ }^{33}$ More recently, Zhang et al demonstrated that DHEA supplementation in patients with poor ovarian response for three IVF cycles (about 3 months) increased DHEAS and total testosterone by 4.4-fold and threefold, respectively. ${ }^{31}$ While their DHEAS response was greater than the current study, we did demonstrate a comparable total testosterone response with very similar baseline and posttreatment testosterone levels. ${ }^{31}$ Interestingly, this group supplemented IVF patients with more DHEA ( $75 \mathrm{mg}$ daily) and for a longer time frame (three cycles) in comparison to our $50 \mathrm{mg}$ DHEA daily supplementation over 4 weeks, and the former study did not 
reveal how the agent was administered (orally, percutaneously, etc). ${ }^{31}$ Taken together, these data reveal that application of our novel oral troches with a lower concentration of DHEA caused a similar increase in DHEAS levels to previous studies utilizing oral DHEA treatments or topical ointments to bypass first-pass metabolism. However, the amount of serum androstenedione and testosterone using our oral troche formulation was greater than these previous studies, indicating a more efficient therapeutic intervention.

It is clear that androgen levels, particularly DHEA/ DHEAS, decrease with advancing age ${ }^{10}$ by approximately $10 \%$ per decade until approximately 80 years of age..$^{2,34,35}$ Interestingly, we found that individual age had no significant influence on baseline serum analyte concentration. All patients in each age category $(<35,35-39$ and $\geq 40$ years $)$ had similar total and free testosterone, androstenedione, and SHBG concentrations. However, there was a decreasing trend in DHEAS level noted for patients 35 years and above, but this was not significantly different from the average DHEAS level for individuals below 35 years. It is likely that the current study would not detect a significant change in baseline DHEAS levels, as the patient cohort was relatively young, with the oldest patient aged 45 years, and limited to 31 participants. Nonetheless, in our cohort, we found that patient age did not influence serum androgen profile following DHEAsupplementation. When patient responses were stratified according to age, the fold increase in serum concentration was similar for all measured analytes and across all age-groups. This indicated that age did not affect the efficiency of the oral troche treatment.

The relationship between BMI and serum DHEA is unclear, as several conflicting reports have been published. ${ }^{36-39}$ However, from the current study, we have added further evidence to demonstrate that BMI plays a significant role in DHEA and androgen metabolism, but only following supplementation, as no statistically significant difference in hormone levels was detected at baseline measurements when female patients were grouped according to BMI, similar to previous studies. ${ }^{36,40}$ However, after DHEA treatment, generally individuals with a lower BMI displayed an attenuated rise in DHEAS and testosterone levels, but a higher androstenedione response. The impact on DHEAS and testosterone may have been a consequence of higher serum SHBG levels found in these patients. Overall, the best responders tended to be patients in the $25.1-30.0 \mathrm{~kg} / \mathrm{m}^{2}$ BMI category. However, there was a marked reduction in DHEAS, androstenedione, and testosterone responses postsupplementation for obese patients $\left(>30.0 \mathrm{~kg} / \mathrm{m}^{2}\right)$. Our results describe increases after
DHEA treatment, but the results were not statistically different from the corresponding baseline values. This implied that patients of excessive body weight either required a greater dose of DHEA to achieve a statistically significant increase in serum hormones from baseline (perhaps due to greater circulatory volume), or that the administered dose may have been biologically diluted within the larger volume of adipose tissue, or finally that there may have been a higher metabolic aromatization of androgens to estrogens, which is expected in obese individuals with higher fat mass and aromatase activity. ${ }^{41,42}$ Experiments to elucidate these hypotheses are currently under way in the authors' laboratory.

This study has demonstrated the impact of our sublingual DHEA troches on serum androgen profile in patients undertaking IVF programs. This method of DHEA administration attains similar degrees of testosterone, DHEAS, and androstenedione enhancement in comparison with other techniques. ${ }^{31-33}$ More importantly, we have shown that BMI significantly influences DHEA uptake and metabolism, and these data suggested that BMI should be carefully considered during dosage calculation to ensure a significant and robust androgen-profile boost in patients undergoing IVF.

\section{Acknowledgments}

The authors wish to thank the Curtin Health Innovation Research Institute - Biosciences, for providing excellent facilities. We also wish to thank all staff at PIVET Medical Centre and all patients who participated in this study.

\section{Author contributions}

Data were measured and extracted by KNK, PMH, JLC, and JLY. DHEA troches were manufactured by NN. KNK was responsible for data analysis and preparation of the first manuscript draft. Senior authors PN and JLY revised the manuscript. All authors contributed toward data analysis, drafting and revising the paper and agree to be accountable for all aspects of the work.

\section{Disclosure}

The authors report no conflicts of interest in this work.

\section{References}

1. Arlt W. Dehydroepiandrosterone and ageing. Best Pract Res Clin Endocrinol Metab. 2004;18(3):363-380.

2. Maggio M, De Vita F, Fisichella A, et al. DHEA and cognitive function in the elderly. $J$ Steroid Biochem Mol Biol. 2015;145: 281-292.

3. Bruder JM, Sobek L, Oettel M. Dehydroepiandrosterone stimulates the estrogen response element. J Steroid Biochem Mol Biol. 1997; 62(5-6):461-466. 
4. Demirgören S, Majewska MD, Spivak CE, London ED. Receptor binding and electrophysiological effects of dehydroepiandrosterone sulfate, an antagonist of the GABA receptor. Neuroscience. 1991; 45(1):127-135.

5. Gleicher N, Barad DH, Kushnir VA, Sen A, Weghofer A. Poor responders and androgen adjuvant treatment: "Still haven't found what I'm looking for ...". Reprod Biomed Online. 2014;29(5):650-652.

6. Rainey WE, Carr BR, Sasano H, Suzuki T, Mason JI. Dissecting human adrenal androgen production. Trends Endocrinol Metab. 2002; 13(6):234-239.

7. Traish AM, Kang HP, Saad F, Guay AT. Dehydroepiandrosterone (DHEA) - a precursor steroid or an active hormone in human physiology. J Sex Med. 2011;8(11):2960-2982; quiz 2983.

8. Warner M, Gustafsson JA. DHEA - a precursor of ER $\beta$ ligands. J Steroid Biochem Mol Biol. 2015;145:245-247.

9. Celec P, Stárka L. Dehydroepiandrosterone - Is the fountain of youth drying out? Physiol Res. 2003;52(4):397-407.

10. Tivesten A, Vandenput L, Carlzon D, et al. Dehydroepiandrosterone and its sulfate predict the 5-year risk of coronary heart disease events in elderly men. $J$ Am Coll Cardiol. 2014;64(17):1801-1810.

11. Valenti G, Ferrucci L, Lauretani F, et al. Dehydroepiandrosterone sulfate and cognitive function in the elderly: the InCHIANTI study. J Endocrinol Invest. 2009;32(9):766-772.

12. Magri F, Cravello L, Barili L, et al. Stress and dementia: the role of the hypothalamic-pituitary-adrenal axis. Aging Clin Exp Res. 2006;18(2): $167-170$.

13. Ohlsson C, Labrie F, Barrett-Connor E, et al. Low serum levels of dehydroepiandrosterone sulfate predict all-cause and cardiovascular mortality in elderly Swedish men. J Clin Endocrinol Metab. 2010; 95(9):4406-4414.

14. Phillips AC, Carroll D, Gale CR, Lord JM, Arlt W, Batty GD. Cortisol, DHEA sulphate, their ratio, and all-cause and cause-specific mortality in the Vietnam Experience Study. Eur J Endocrinol. 2010;163(2): 285-292.

15. Tchernof A, Després JP, Bélanger A, et al. Reduced testosterone and adrenal C19 steroid levels in obese men. Metabolism. 1995;44(4): 513-519.

16. Deighton CM, Watson MJ, Walker DJ. Sex hormones in postmenopausal HLA-identical rheumatoid arthritis discordant sibling pairs J Rheumatol. 1992;19(11):1663-1667.

17. Mazat L, Lafont S, Berr C, et al. Prospective measurements of dehydroepiandrosterone sulfate in a cohort of elderly subjects: relationship to gender, subjective health, smoking habits, and 10-year mortality. Proc Natl Acad Sci U S A. 2001;98(14):8145-8150.

18. Ding EL, Song Y, Manson JE, Rifai N, Buring JE, Liu S. Plasma sex steroid hormones and risk of developing type 2 diabetes in women: a prospective study. Diabetologia. 2007;50(10):2076-2084.

19. Narkwichean A, Maalouf W, Campbell B, Jayaprakasan K. Efficacy of dehydroepiandrosterone to improve ovarian response in women with diminished ovarian reserve: ameta-analysis. Reprod BiolEndocrinol.2013; 11:44.

20. Weil S, Vendola K, Zhou J, Bondy CA. Androgen and follicle-stimulating hormone interactions in primate ovarian follicle development. $J$ Clin Endocrinol Metab. 1999;84(8):2951-2956.

21. Pitteloud N, Mootha VK, Dwyer AA, et al. Relationship between testosterone levels, insulin sensitivity, and mitochondrial function in men. Diabetes Care. 2005;28(7):1636-1642.

22. Sunkara SK, Pundir J, Khalaf Y. Effect of androgen supplementation or modulation on ovarian stimulation outcome in poor responders: a meta-analysis. Reprod Biomed Online. 2011;22(6):545-555.

23. Ferraretti AP, La Marca A, Fauser BC, et al. ESHRE consensus on the definition of 'poor response' to ovarian stimulation for in vitro fertilization: the Bologna criteria. Hum Reprod. 2011;26(7):1616-1624.

24. Gleicher N, Barad D. Dehydroepiandrosterone (DHEA) supplementation in diminished ovarian reserve (DOR). Reprod Biol Endocrinol. 2011; 9:1-12.
25. Casson PR, Lindsay MS, Pisarska MD, Carson SA, Buster JE. Dehydroepiandrosterone supplementation augments ovarian stimulation in poor responders: a case series. Hum Reprod. 2000;15(10): 2129-2132.

26. Barad D, Gleicher N. Effect of dehydroepiandrosterone on oocyte and embryo yields, embryo grade and cell number in IVF. Hum Reprod. 2006;21(11):2845-2849.

27. Barad D, Brill H, Gleicher N. Update on the use of dehydroepiandrosterone supplementation among women with diminished ovarian function. J Assist Reprod Genet. 2007;24(12):629-634.

28. Gleicher N, Weghofer A, Barad D. Dehydroepiandrosterone (DHEA) reduces embryo aneuploidy: direct evidence from preimplantation genetic screening (PGS). Reprod Biol Endocrinol. 2010;8:140.

29. Wiser A, Gonen O, Ghetler Y, Shavit T, Berkovitz A, Shulman A. Addition of dehydroepiandrosterone (DHEA) for poor-responder patients before and during IVF treatment improves the pregnancy rate: a randomized prospective study. Hum Reprod. 2010;25(10): 2496-2500.

30. Xu B, Li Z, Yue J, et al. Effect of dehydroepiandrosterone administration in patients with poor ovarian response according to the Bologna criteria. PLoS One. 2014;9(6):e99858.

31. Zhang HH, Xu PY, Wu J, et al. Dehydroepiandrosterone improves follicular fluid bone morphogenetic protein-15 and accumulated embryo score of infertility patients with diminished ovarian reserve undergoing in vitro fertilization: a randomized controlled trial. J Ovarian Res. 2014;7:93

32. Morales AJ, Nolan JJ, Nelson JC, Yen SS. Effects of replacement dose of dehydroepiandrosterone in men and women of advancing age. J Clin Endocrinol Metab. 1994;78(6):1360-1367.

33. Labrie F, Bélanger A, Cusan L, Candas B. Physiological changes in dehydroepiandrosterone are not reflected by serum levels of active androgens and estrogens but of their metabolites: intracrinology. J Clin Endocrinol Metab. 1997;82(8):2403-2409.

34. Havelock JC, Auchus RJ, Rainey WE. The rise in adrenal androgen biosynthesis: adrenarche. Semin Reprod Med. 2004;22(4): 337-347.

35. Orentreich N, Brind JL, Rizer RL, Vogelman JH. Age changes and sex differences in serum dehydroepiandrosterone sulfate concentrations throughout adulthood. J Clin Endocrinol Metab. 1984;59(3): 551-555.

36. Kraemer RR, Synovitz LB, Gimpel T, Kraemer GR, Johnson LG, Castracane VD. Effect of estrogen on serum DHEA in younger and older women and the relationship of DHEA to adiposity and gender. Metabolism. 2001;50(4):488-493.

37. Voznesensky M, Walsh S, Dauser D, Brindisi J, Kenny AM. The association between dehydroepiandosterone and frailty in older men and women. Age Ageing. 2009;38(4):401-406.

38. Barrett-Connor E, Ferrara A. Dehydroepiandrosterone, dehydroepiandrosterone sulfate, obesity, waist-hip ratio, and noninsulin-dependent diabetes in postmenopausal women: the Rancho Bernardo study. J Clin Endocrinol Metab. 1996;81(1):59-64.

39. Hsieh CC, Signorello LB, Lipworth L, Lagiou P, Mantzoros CS, Trichopoulos D. Predictors of sex hormone levels among the elderly: a study in Greece. J Clin Epidemiol. 1998;51(10):837-841.

40. Kumar A, Woods KS, Bartolucci AA, Azziz R. Prevalence of adrenal androgen excess in patients with the polycystic ovary syndrome (PCOS) Clin Endocrinol. 2005;62(6):644-649.

41. Jasik CB, Lustig RH. Adolescent obesity and puberty: the "perfect storm". Ann N Y Acad Sci. 2008;1135:265-279.

42. Bélanger C, Luu-The V, Dupont P, Tchernof A. Adipose tissue intracrinology: potential importance of local androgen/estrogen metabolism in the regulation of adiposity. Horm Metab Res. 2002;34(11-12): $737-745$. 


\section{Publish your work in this journal}

Drug Design, Development and Therapy is an international, peerreviewed open-access journal that spans the spectrum of drug design and development through to clinical applications. Clinical outcomes, patient safety, and programs for the development and effective, safe, and sustained use of medicines are a feature of the journal, which

has also been accepted for indexing on PubMed Central. The manuscript management system is completely online and includes a very quick and fair peer-review system, which is all easy to use. Visit http://www.dovepress.com/testimonials.php to read real quotes from published authors.

Submit your manuscript here: http://www.dovepress.com/drug-design-development-and-therapy-journal 